Mobility and health

\section{Assessing the impact of mobility on health: implications for life course epidemiology}

\section{Alastair H Leyland}

\section{Future research should consider the interaction between length of exposure to an area or household and the stage of life course.}

T he need to use multilevel models when analysing hierarchical data-

to take account of the correlation in the data when estimating regression coefficients-is widely recognised in health research. ${ }^{12}$ The advantages afforded by multilevel models-including the ability to partition variation to determine the relative importance of different levels of the hierarchy, to test hypotheses about variation, and to attempt to separate the influences of context and composition-are also well reported. ${ }^{3}$

The complexity of the real world means that our data are not always drawn from strict hierarchies. ${ }^{4}$ In this issue of the journal Chandola et al consider a multiple membership model - a situation in which individuals may belong to more than one unit at a higher level. ${ }^{5}$ Their data follow up individuals over nine years during which time they may belong to several households and may move from one area to another. However, it is only data on residence that are longitudinal; data on health are restricted to the end of the study. This editorial considers how multilevel modelling can be used to analyse such data to give an insight into contextual influences at different stages of the life course.

Consider a two level model of individuals in areas. If the data were strictly hierarchical (or equivalently, in this case, if they were cross sectional) the health of the individual might be modelled in terms of the known characteristics of the individual and area plus unexplained effects (or random effects $^{6}$ ) at each level. But if people move from one area to another andthrough whatever mechanism-areas have an effect on individual health, we might expect the total effect of place on an individual's health to be an accumulation of the effects of all the areas in which that person resided. Figure 1A shows how an individual $P_{1}$ might be living in area $A_{1}$ at time $T_{1}$ but in area $A_{2}$ at time $T_{2}$. Denoting the effects for the two areas by $u_{1}$ and $u_{2}$ respectively, the cumulative area effects on those who did not move- $-P_{2}$ and $P_{3}$-would be $u_{1}$ and $u_{2}$ respectively. For individual $P_{1}$, having spent equal amounts of time in each area, the net contribution to health would be $1 / 2 u_{1}+$ $1 / 2 u_{2}$.

This suggests that the cumulative health effect of area of residence on $P_{1}$ lies halfway between the effects on people who had spent all their life in areas $A_{1}$ and $A_{2}$ - individuals $P_{2}$ and $P_{3}$. This is a pleasing result and clearly is of potential importance for contextual influences in life course epidemiology. However, its impact will be limited for two reasons. Firstly, there is no notion that places may change over time; the effect of area $A_{1}$ at times $T_{1}$ and $T_{2}$ is assumed to be constant, $u_{1}$. Secondly, the direction of movement is not taken
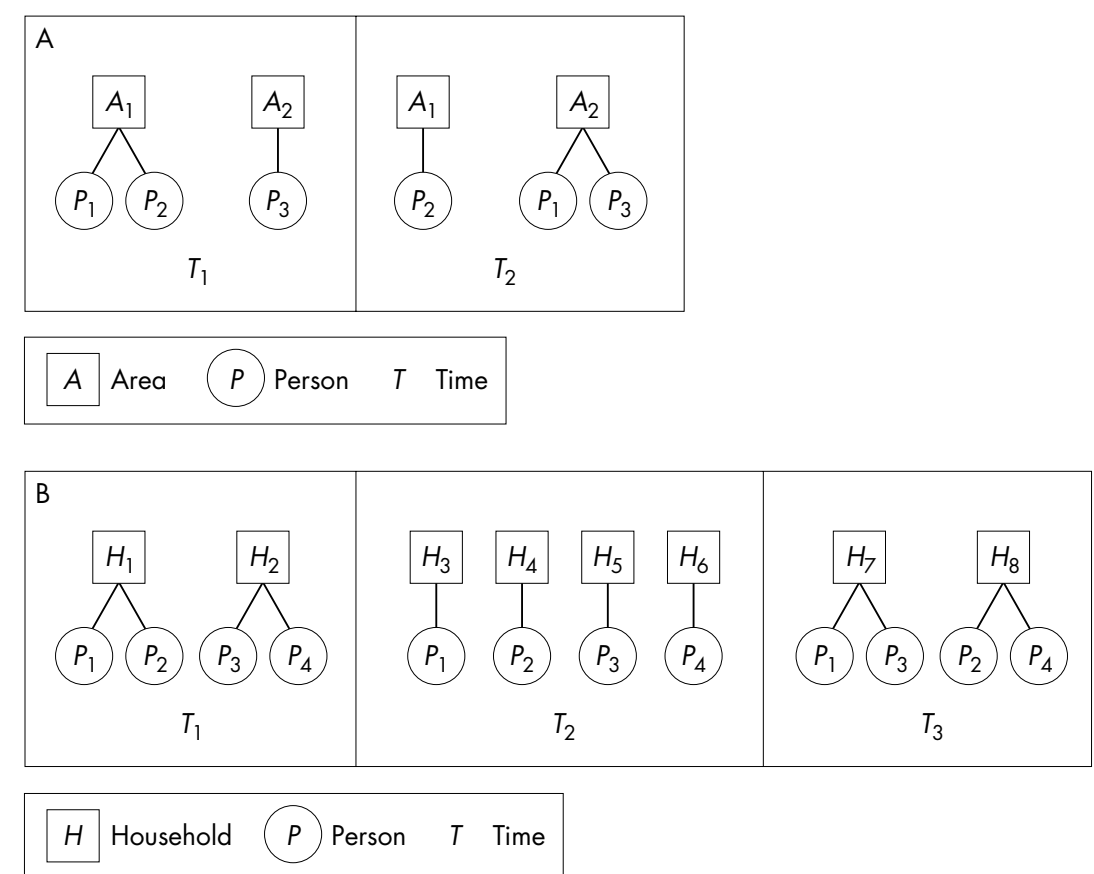

Figure 1 Illustration of changing group membership over time. (A) Moving from one area to another; (B) changing household dynamics.

$$
\operatorname{Var}\left(1 / 2 \mathcal{u}_{1}+1 / 2 \mathcal{u}_{2}\right)=1 / 2 \sigma_{u}^{2}
$$
individual level. into account; if area $A_{2}$ is the most recently inhabited then the magnitude of its influence may differ from that of $A_{1}$. And in life course epidemiology this could be crucial-the difference between the area of residence in childhood and in old age, for example.

There is also a mathematical consequence of these models for the area variances. For a static individual such as $P_{2}$ the total variance arising from the area of residence is

$$
\operatorname{Var}\left(u_{1}\right)=\sigma_{u}^{2}
$$

while for a mobile individual such as $P_{1}$ the corresponding variance is

As a mean, the variance of the cumulative health effect of area of residence for mobile individuals is always lower than for static individuals. But is there less variability in the health of mobile than static individuals? If not then the estimate of the variance between areas $\sigma_{u}^{2}$ will be inflated unless the additional variability in the health of mobile individuals is modelled at the

When an individual moves from one area to another it is easy to think of the area as remaining comparatively unchanged as one person is unlikely to affect the area's defining character or its influence on health, but what of the household? With such a small unit one 
person joining or leaving may change the household dynamics and its effect on the individuals' health. It may be that little remains constant beside the physical environment (if the new household lives in the same physical dwelling). Figure 1B illustrates how households may evolve over time. At time $T_{1}$ there are two households $-H_{1}$ and $\mathrm{H}_{2}$-each containing two people $P_{1}-P_{4}$. By time $T_{2}$ both households have broken up, forming four new one person households, and by time $T_{3}$ two new households have formed containing different combinations of the same four people. It is difficult to think of any of the six subsequent households $H_{3}-H_{8}$ as having much in common with $H_{1}$ and $\mathrm{H}_{2}$. Cross sectional studies conducted at two time points would find the same areas but different households; this means that it is not possible to examine the effect of household membership at different time points in the same way as can be done for areas. The debate as to the extent to whether differences between areas reflect differences in contexts as compared with differences in their composition ${ }^{8}$ is even more relevant to the small household unit: is a household any more than the sum of its constituent members? Are these household effects or interactions between individuals?

Certainly household and area remain important units for analysis in our attempt to understand individuals' health and health behaviours. The paper by Chandola et al extends this by showing the potential importance of taking changing membership into account and the technique by which we might do this. Their methodology takes account of length of exposure to the area or household during the study period; future research must consider the interaction between the exposure and the stage of the life course.

$J$ Epidemiol Community Health 2005;59:90-91.

doi: $10.1136 /$ jech. 2004.027466

Correspondence to: $\operatorname{Dr} \mathrm{A} H$ Leyland, MRC Social and Public Health Sciences Unit, University of Glasgow, 4 Lilybank Gardens, Glasgow G12 8RZ, Scotland; a.leyland@msoc. mrc.gla.ac.uk
Funding: the Social and Public Health Sciences Unit is jointly funded by the Medical Research Council and the Chief Scientist Office of the Scottish Executive Health Department.

Competing interests: none declared.

\section{REFERENCES}

1 Diez Roux AV. Multilevel analysis in public health research. Annu Rev Public Health 2000;21:171-92.

2 Leyland AH, Goldstein $\mathrm{H}$. Multilevel modelling of health statistics. Chichester: Wiley, 2001.

3 Leyland AH, Groenewegen PP. Multileve modelling and public health policy. Scand J Public Health 2003;31:267-74.

4 Rasbash J, Browne W. Modelling nonhierarchical structures. In: Leyland $\mathrm{AH}$, Goldstein $\mathrm{H}$, eds. Multilevel modelling of health statistics. Chichester: Wiley, 2001:93-105.

5 Chandola T, Clarke P, Wiggins RD, et al. Who you live with and where you live: setting the context for health using multiple membership multilevel models. J Epidemiol Community Health 2005;59:170-5.

6 Diez Roux AV. A glossary for multilevel analysis. J Epidemiol Community Health 2002;56:588-94.

7 Macintyre S, Maclver S, Sooman A. Area, class and health: should we be focusing on places or people? Journal of Social Policy 1993;22:213-34.

8 Sloggett A, Joshi $\mathrm{H}$. Higher mortality in deprived areas: community or personal disadvantage? BMJ 1994;309:1470-4.

\section{THE JECH GALLERY}

\section{"Low-Carb": America's latest food fad}

\begin{abstract}
A ny visitor to the USA cannot help but notice the current fixation with minimising (or even eradicating) carbohydrates from the American diet. Advertisements proffer all manner of lowcarb options (for example, bread, pizza, chocolate). There are low-carb specialty shops and menus in restaurants offer lowcarb dishes. You can even wash down you low-carb meal with low-carb wine (see left hand image). This contrasts with the situation in England, where the Atkins inspired diet has arrived but has yet to reach the same scale of acceptance (see right hand image). It will be interesting to see if this new fad will have a similar effect on American waistlines as low calorie, low fat obsessions of past decades.
\end{abstract}

Mary Shaw

Department of Social Medicine, University of Bristol, Canynge Hall, Whiteladies Road, Bristol BS8 2PR, and South West Public Health

Observatory, UK; mary.shaw@bristol.ac.uk

Funding: Mary Shaw is funded by the South West Public Health Observatory
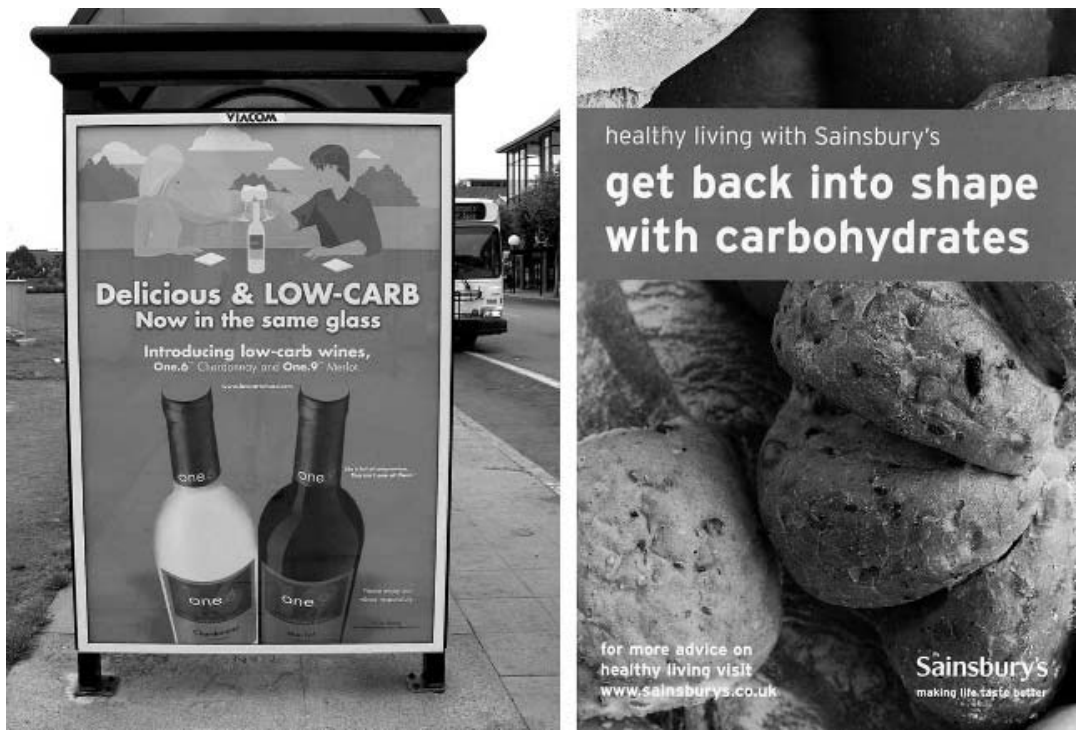

Left: an advertisement in San Francisco, USA, August 2004 (taken by Mary Shaw); right: a supermarket leaflet, England, August 2004. 